\title{
AVALIAÇÃO DO RISCO DE QUEDAS DE PACIENTES EM SERVIÇO DE EMERGÊNCIA
}

\section{EVALUATION OF THE RISK OF FALLS OF PATIENTS IN EMERGENCY SERVICE}

\section{EVALUACIÓN DEL RIESGO DE CUIDAS DE PACIENTES EN SERVICIO DE EMERGENCIA}

\author{
Paloma Horbach da Rosa ${ }^{1}$, Rosiane Filipin Rangel ${ }^{2}$, Karine de Freitas Cáceres Machado ${ }^{3}$, Mariana Pellegrini Cesar ${ }^{4}$, Silomar llha ${ }^{5}$.
}

\begin{abstract}
RESUMO
Objetivo: Identificar o risco de quedas nos pacientes em observação em um serviço de emergência, por meio da aplicação da Escala de Quedas de Morse. Métodos: Estudo quantitativo, com dados transversais do tipo descritivo, desenvolvido com 30 pacientes internados em um serviço de emergência do Rio Grande do Sul. Os dados foram coletados, entre junho e julho/2018, pela aplicação da Escala de Quedas de Morse. Nas primeiras 12 horas de internação os pacientes foram analisados pela estatística descritiva simples. Resultados: Dos 30 pacientes, 15 eram do sexo feminino e 15 do masculino, com idade entre 30 e 83 anos. Destes, 16 $(53,33 \%)$ apresentaram alto, 07 (23,33\%) médio e 07 (23,33\%) baixo risco para quedas. Os fatores de risco mais encontrados foram: terapia endovenosa, diagnóstico secundário e marcha. Conclusão: Os dados representam um alerta aos enfermeiros para o diagnóstico precoce do risco de quedas, prescrição e implementação dos cuidados pela equipe de enfermagem. Assim como a necessidade de implantação de protocolos de prevenção de quedas no serviço de emergência e a realização da avaliação do risco de quedas, diariamente, pelo enfermeiro como uma ferramenta para garantir um cuidado seguro.
\end{abstract}

Descritores: Acidentes por Quedas; Emergências; Pesos e medidas; Enfermagem.

\section{ABSTRACT}

Objective: To identify the risk of falls in patients under observation in an emergency service, through the application of the Morse Falls Scale. Methods: A quantitative study with descriptive cross-sectional data was developed with 30 patients in emergency service in Rio Grande do Sul. Data were collected from June to July 2018. In the first 12 hours of hospitalization, patients were analyzed by simple descriptive statistics. Results: Of the 30 patients, 15 were female and 15 male, aged between 30 and 83 years. A total of 16 patients $(53.33 \%)$ presented a high risk of falling, while 07 (23.33\%) presented medium risk and 07 presented $(23.33 \%)$ low risk. The most common risk factors were: intravenous therapy, secondary diagnosis, and gait. Conclusion: The results represent an alert to nurses for the early diagnosis of the risk of falls, prescription, and implementation of care by the nursing team. Furthermore, there is a need to implement protocols for the prevention of falls in the emergency service and to perform fall risk assessments by nurses on a daily basis as a tool to ensure safe care.

Descriptors: Accidental Falls; Medical Emergencies; Weights and Measures; Nursing.

\section{RESUMEN}

Objetivo: Identificar el riesgo de caídas en los pacientes en observación en un servicio de emergencia, por medio de la aplicación de la Escala de Caídas de Morse. Métodos: Estudio cualitativo, descriptivo, desarrollado con 30 pacientes internados en un servicio de emergencia de Rio Grade del Sur. Datos recolectados entre junio y julio de 2018, por medio de la aplicación de la Escala de Caídas de Morse. Las primeras 12 horas de internación de los pacientes fueron analizadas por la estadística descriptiva simple. Resultados: De los 30 pacientes, 15 eran del sexo femenino y 15, masculino, con edad entre 30 y 83 años. De estos, 16 (53,33\%) presentaron alto, $07(23,33 \%)$ promedio y $07(23,33 \%)$, bajo riesgo para caídas. Los factores de riesgo más encontrados fueron: terapia endovenosa, diagnóstico secundario y marcha. Conclusión: Los datos representan una alerta a los enfermeros para el diagnóstico precoz del riesgo de caídas, prescripción e implementación de los cuidados por el equipo de enfermería. Así como la necesidad de implantación de protocolos de prevención de caídas en el servicio de emergencia y la realización de la evaluación del riesgo de caídas, a diario, por el enfermero como una herramienta para garantizar un cuidado seguro.

Descriptores: Accidentes por Caídas; Emergencias; Pesos y Medidas; Enfermería.

${ }^{1}$ Especialista em Urgência-Trauma, Mestranda na Universidade Federal de Santa Maria - UFSM. ${ }^{2}$ Doutora em Enfermagem, Docente da Universidade Franciscana. ${ }^{3}$ Mestre em Enfermagem, Docente da Universidade Franciscana. ${ }^{4}$ Especialista em Urgência-Trauma, Mestranda da UFSM. ${ }^{5}$ Doutor em Enfermagem, Docente da Universidade Franciscana. 


\section{INTRODUÇÃO}

A Organização Mundial de Saúde (OMS) tem desenvolvido ações com a finalidade de proteger a saúde da população e intervir nos riscos advindos do uso de produtos e dos serviços a ela sujeitos. Em busca de garantir a segurança do paciente e prestar uma assistência qualificada, utiliza-se de práticas de vigilância, controle, regulação e monitoramento sobre os serviços de saúde e o uso das tecnologias disponíveis para o cuidado $^{(1)}$.

Nesse contexto, a Aliança Mundial para a Segurança do Paciente divulgou, durante os anos de 2008 e 2009, as novas diretrizes para a segurança do paciente, as quais traduzem os conhecimentos científicos em soluções práticas para serem difundidas, mundialmente, com vistas a minimizar os eventos adversos. Entre essas, encontram-se a identificação do paciente; a comunicação durante a passagem de plantão e transferência do paciente; a realização de procedimento correto, no local correto; o controle de soluções concentradas de eletrólitos; a segurança na medicação nas transições de cuidado, durante a passagem de plantão; conexões corretas entre cateteres e sondas e a prevenção de quedas ${ }^{(2)}$.

Os eventos adversos são caracterizados como incidentes indesejáveis, porém preveníveis, que ocorrem durante a assistência à saúde. Esses podem gerar danos na estrutura ou função do corpo, ou efeito nocivo como doença, lesão, incapacidade e, até mesmo, a morte, podendo ser de caráter físico, social e/ou psíquico ${ }^{(3)}$.

Dentre os principais eventos adversos a serem prevenidos em instituições de saúde, destaca-se a redução do risco de quedas. Por queda, compreende-se um contato não intencional com a superfície de apoio em consequência da mudança de posição da pessoa para um nível inferior à sua posição inicial, sem que tenha fator intrínseco determinante ${ }^{(4)}$.

As quedas em pacientes hospitalizados causam inúmeras consequências imediatas para a pessoa que a sofreu. Além dos danos teciduais, são citadas como consequências no aumento do tempo de hospitalização, alto custo de tratamento e descrença do paciente em relação aos cuidados de enfermagem ${ }^{(5)}$.

A avaliação do paciente e a identificação das características que podem aumentar a probabilidade de quedas tornam-se fundamentais para o planejamento de estratégias de prevenção efetivas no cenário hospitalar. Assim, a utilização de ferramentas específicas na identificação de indivíduos com maior suscetibilidade de cair pode ser uma aliada na prevenção do incidente ${ }^{(6)}$.

Existem pesquisas relacionadas a quedas em diferentes cenários, entretanto, no Brasil, existe uma lacuna relacionada a estudos que investiguem a incidência desse evento em ambiente de emergência, bem como da avaliação do risco, por meio de instrumentos validados. Dentre estes, encontra-se a Morse Fall Scalepor (MFS), que permite efetivamente identificar o risco de quedas em adultos que se encontram internados. Caracteriza-se como uma escala traduzida e adaptada transculturalmente para a língua portuguesa e mundialmente utilizada ${ }^{(7)}$.

A avaliação do paciente para o risco de quedas, tanto no momento de sua admissão na instituição de saúde quanto no transcorrer da internação deve ser realizada, uma vez que se torna uma importante ferramenta de cuidado. Dessa forma, permite que, os profissionais de saúde, especialmente os enfermeiros, por serem os responsáveis pela sistematização do cuidado, implementam os cuidados necessários para a prevenção de eventos adversos ${ }^{(1)}$.

Considerando os agravos que as quedas podem ocasionar ao paciente, à instituição e para a equipe de saúde, apresenta-se a seguinte pergunta de pesquisa: quais são os fatores de risco relacionados a quedas, encontrados em um serviço de emergência? Diante desse questionamento, este estudo teve por objetivo identificar o risco de quedas nos pacientes em observação em um serviço de emergência, por meio da aplicação da Escala de Quedas de Morse.

\section{MÉTODO}

Estudo transversal do tipo descritivo ${ }^{(8-9)}$, desenvolvido em um serviço de emergência da região central, do Rio Grande do Sul, cuja coleta de dados ocorreu do período de junho e julho de 2018.

Para seleção dos sujeitos desta pesquisa foi utilizada amostragem aleatória, tendo como critérios de inclusão pacientes maiores de 18 anos em leitos de observação adulta, com até 12 horas em observação.

A escolha por esse critério de tempo ocorreu pelo fato de que a MFS tem indicação de ser aplicada no momento da admissão do paciente e, diariamente, enquanto estiver no ambiente hospitalar ou ainda, a cada alteração das condições clínicas. Nesse sentido, pacientes 
internados a mais de 12 horas impossibilitariam a dinâmica de reavaliação das situações e riscos.

Foram excluídos do estudo pacientes que não tivessem capacidade cognitiva preservada para autorizar a realização da pesquisa. Para avaliação cognitiva seletiva dos participantes, foi utilizado o instrumento denominado Miniexame do Estado Mental (MEEM), que é um instrumento de avaliação cognitiva e rastreamento de quadros demenciais ${ }^{(10)}$.

A amostra aleatória justifica-se pela necessidade de definir o perfil dos pacientes que procuram o serviço de emergência e os fatores de risco para a ocorrência das quedas. A coleta de dados foi realizada, através da aplicação da MFS junto ao paciente, nas primeiras $12 \mathrm{~h}$ de sua observação. $\mathrm{O}$ número da amostra $(\mathrm{N})$ foi de 30 participantes. A análise dos dados foi realizada, por meio de estatística descritiva simples, sustentada pelas publicações nacionais e internacionais, assim como o referencial de segurança do paciente na discussão dos dados apresentados.

O serviço de emergência, cenário da coleta de dados deste estudo, dispõe de 12 leitos de observação, sendo eles masculinos ou femininos, dependendo da demanda. Entretanto, devido à fragilidade de encaminhamentos para os serviços de referências da região de saúde correspondente, em consequência da alta demanda, os pacientes permanecem por um período superior às 12 horas de observação. Por vezes, permanecem no serviço de emergência realizando o tratamento necessário, até a alta. Ainda, no período de coleta de dados, o referido serviço, passou por processo de reforma estrutural, disponibilizando de apenas três leitos de observação, diminuindo ainda mais a rotatividade dos leitos.

A MFS possui seis itens com pontuações diferentes entre eles, podendo variar de 0 a 125 pontos. 0 paciente classificado entre 0 a 24 pontos possui baixo risco de queda; o paciente classificado 25 a 44 pontos possui risco moderado de queda; e os pacientes com 45 pontos ou mais possuem alto risco de queda ${ }^{(7)}$.

Os instrumentos de coleta de dados foram aplicados de forma sequencial, sendo o MEEM, a aplicação da MFS e, por fim, a análise do prontuário. Esses instrumentos foram aplicados no serviço de emergência, individualmente, no leito, após a explanação sobre a pesquisa e assinatura do Termo de Consentimentos Livre e Esclarecido (TCLE), em duas vias. O histórico de queda foi autorreferido pelo paciente, a presença de diagnóstico secundário e a utilização de terapia endovenosa foi confirmado através do prontuário, o auxílio na deambulação, alterações na marcha e avaliação do estado mental foram avaliadas com base na definição operacional da Escala de Morse $^{(7)}$.

A pesquisa foi orientada pela Resolução $n$ ㅇ 466/12, do Ministério da Saúde ${ }^{(11)}$. A coleta de dados teve inicio após a aprovação da instituição e CEP/UFN, sob o parecer no 2.643 .103 , de oito de maio de 2018.

\section{RESULTADOS E DISCUSSÃO}

Participaram do estudo 30 pacientes, dos quais, 15 do sexo feminino e 15 do sexo masculino, com idade entre 30 e 83 anos, 56,67\% dos pacientes com idade maior de 60 anos (Tabela 1).

Tabela 1 - Caracterização sociodemográficas dos pacientes em observação em serviço de emergência - Rio Grande do Sul, Brasil, 2018.

\begin{tabular}{lc} 
& $\%$ \\
Sexo & \\
Feminino & $50,00 \%$ \\
Masculino & $50,00 \%$ \\
Idade & \\
Menores de 60 anos & $43,33 \%$ \\
Maiores de 60 anos & $56,67 \%$ \\
\hline
\end{tabular}

Fonte: dados da investigação, 2018.

Pacientes maiores de 60 anos apresentaram alto risco queda (70,59\%), conforme a MFS, quando comparados aos pacientes menores de 60 anos. Estudo realizado em departamento de emergência corrobora com os dados encontrados neste estudo ${ }^{(12)}$, apresentando também, alto índice de risco de queda.

A idade acima de 60 anos é considerada como maior fator de risco de queda e para as lesões decorrentes desse evento. Esse fato é explicado pelas alterações fisiológicas do próprio 
envelhecimento, que são preditoras de quedas, tais como problemas na mobilidade física, incluindo a instabilidade postural e alteração de marcha, diminuição da capacidade cognitiva, funcional e visual. Outros fatores associados são as doenças crônicas degenerativas e a polifarmácia que são condições comuns nos idosos e que também podem aumentar o risco de queda ${ }^{(13)}$.

Observou-se que a maioria dos pacientes apresentava ensino fundamental incompleto
(60,00\%). Estudo indica que o nível educacional influencia na localização espacial, de modo que, ao executar tarefas de busca, pessoas com baixo nível de escolaridade necessitam de mais tempo e erram mais ao realizar as tarefas ${ }^{(14)}$, o que pode aumentar o risco de quedas.

O resultado do estudo apontou que dos 30 pacientes avaliados, $16(53,33 \%)$ apresentaram alto risco para quedas, 07 (23,33\%) apresentaram risco médio e 07 (23,33\%) apresentaram baixo risco, de acordo com a MFS (Tabela 2).

Tabela 2 - Classificação dos pacientes quanto ao risco de queda pela Escala de Morse, durante o período de observação - Rio Grande do Sul, Brasil, 2018.

\begin{tabular}{|c|c|c|}
\hline \multicolumn{3}{|l|}{ Histórico de Quedas } \\
\hline Sim & 10 & $33,33 \%$ \\
\hline Não & 20 & $66,67 \%$ \\
\hline \multicolumn{3}{|l|}{ Diagnóstico Secundário } \\
\hline Sim & 20 & $66,67 \%$ \\
\hline Não & 10 & $33,33 \%$ \\
\hline \multicolumn{3}{|l|}{ Auxílio na deambulação } \\
\hline Nenhum/acamado/auxiliado por profissional de saúde & 24 & $80,00 \%$ \\
\hline Muletas/Bengala/Andador & 03 & $10,00 \%$ \\
\hline Mobiliário/Andador & 03 & $10,00 \%$ \\
\hline \multicolumn{3}{|l|}{ Terapia Endovenosa } \\
\hline Sim & 26 & $86,67 \%$ \\
\hline Não & 04 & $13,33 \%$ \\
\hline \multicolumn{3}{|l|}{ Marcha } \\
\hline Normal & 20 & $66,67 \%$ \\
\hline Fraca & 04 & $13,33 \%$ \\
\hline Comprometida/cambaleante & 06 & $20,00 \%$ \\
\hline \multicolumn{3}{|l|}{ Estado Mental } \\
\hline Orientado/capaz quanto à sua capacidade/limitação & 25 & $83,33 \%$ \\
\hline Superestima capacidade/Esquece limitações & 05 & $16,67 \%$ \\
\hline \multicolumn{3}{|l|}{ Morse Escore* } \\
\hline Baixo risco & 07 & $23,33 \%$ \\
\hline Médio risco & 07 & $23,33 \%$ \\
\hline Alto risco & 16 & $53,33 \%$ \\
\hline Total & 30 & $100 \%$ \\
\hline
\end{tabular}

Fonte: dados da investigação, 2018.

*Notas: Pontuação da escala: 0-24, baixo risco; 25-44, médio risco; > 45 risco elevado.

O estudo em questão aponta que o setor de emergência apresenta um elevado número de pacientes com alto risco para queda $(53,33 \%)$. Estudo semelhante traz que, o ambiente de emergência apresenta características específicas, tais como: corredores lotados, espaço reduzido, alto fluxo de pessoal que, por sua vez, aumentam o risco de queda ${ }^{(12)}$,ainda, os pacientes permanecem nesses serviços por períodos maiores e exigem maior complexidade na assistência. Contudo, apesar do elevado índice de queda em serviços de emergência, existem poucos estudos que abordem a ocorrência de quedas, nesses serviços, apresentando maior número de estudos realizados em unidades de internação ${ }^{(12)}$.
Nesse contexto, algumas particularidades dos serviços de emergência podem atribuir o alto risco de quedas aos pacientes, posto que, esses setores podem apresentar inadequações de equipamentos assistenciais e estrutura física ${ }^{(15)}$. Além do mais, as macas utilizadas pelos pacientes, por vezes, são estreitas e altas e, geralmente, não possuem grades de proteção ${ }^{(15)}$. A sobrecarga da equipe de enfermagem é apontada como um dos fatores de risco para ocorrência de evento adverso, podendo influenciar, negativamente, na qualidade da assistência à saúde prestada ${ }^{(16)}$.

Pelo fato de o serviço não apresentar um protocolo de avaliação e prevenção da ocorrência de quedas, dificulta o planejamento do cuidado de enfermagem, produzindo multiplicidade de 
informações e não identificação dos riscos existentes. Nesse aspecto, o Ministério da Saúde (MS) em colaboração com a Agência Nacional de Vigilância Sanitária (ANVISA) e com a Fundação FioCruz, elaborou um protocolo de prevenção de quedas pelo Programa Nacional de Segurança do Paciente, com a finalidade de reduzir a ocorrência de quedas e o dano das mesmas, aos pacientes ${ }^{(1)}$. Nesse ínterim, percebeu-se que a instituição estudada não apresentava um protocolo de avaliação e prevenção de quedas, o que existia eram iniciativas no Núcleo de Segurança do Paciente (NSP) de implementação da avaliação do risco de quedas, através da MFS e a notificação de eventos adversos. Com o apoio dessas informações, o enfermeiro responsável adotava as devidas medidas de segurança e estratégias de prevenção.

Pacientes com história de quedas anteriores apresentam maior probabilidade de apresentar nova queda ao desenvolver atividade semelhante a que o fez cair anteriormente. Considerando o exposto, com a obtenção de informações relativas ao histórico de quedas, o enfermeiro pode implementar medidas de prevenção ${ }^{(17)}$. Em relação à variável de Histórico de Quedas, 10 (33,33\%) da população estudada apresentaram queda nos últimos três meses, enquanto $20(66,67 \%)$ não apresentaram risco nesse fator. Em outro estudo, também identificou-se, na admissão do paciente, que $30,3 \%$ dos pacientes tinham histórico de queda anterior $^{(18)}$.

Outro fator de risco que está intimamente relacionado à ocorrência de quedas é a presença de Diagnóstico Secundário, observada em 66,67\% dos pacientes, ou seja, 20 pacientes apresentaram um ou mais diagnósticos, além do motivo da internação, sendo o segundo fator mais prevalente, corroborando com outro estudo que apresentou maior percentual de pacientes com diagnóstico secundário ${ }^{(6)}$. A instituição deste estudo assiste a uma grande demanda de idosos com múltiplas doenças e, assim, ratifica a maior propensão a quedas. No que concerne ao Auxílio na Deambulação, 06 (20,00\%) pacientes apresentaram esse fator de risco, sendo que destes, $03(10,00 \%)$ utilizavam cadeira de rodas, bengalas ou andador e 03 (10,00\%) buscavam apoio em mobiliário.

Observou-se que, quando questionados sobre a maneira como se locomoviam pelo setor, $20 \%$ dos pacientes responderam que necessitavam de auxílio para deambular. Porém, quando avaliados pelo enfermeiro em relação à marcha, 33,33\% dos pacientes apresentaram algum tipo de alteração, sendo ela fraca ou comprometida.

Esses dados evidenciam a importância do enfermeiro direcionar a atenção na avaliação inicial do paciente, visto que algumas vezes esse aspecto é subnotificado ou não avaliado, adequadamente, sendo que a minoria dos pacientes relata a necessidade de auxílio para deambular. Reconhecendo esse risco antecipadamente, o enfermeiro poderá prescrever cuidados adequados, o que auxiliará na prevenção da ocorrência de quedas.

Pela especificidade do atendimento em situações agudas, como é caracterizado o serviço de emergência, os pacientes podem passar por períodos de incapacidade de locomoção, ou seja, pacientes antes independentes podem tornar-se pacientes dependentes temporariamente. Estudo $^{(12)}$ que realizou análise retrospectiva em prontuários, indicou que há um grande número de pacientes que se locomovem pelo setor, entre área de procedimentos e banheiros, esses pacientes apresentam alto risco para quedas. Nesse momento, a orientação e o auxílio imediato são imprescindíveis para que o paciente não tente se levantar e a deambular sozinho, expondo-se ao risco de queda.

A utilização de terapia endovenosa para hidratação e medicação é considerada um fator de risco às quedas e, neste estudo, foi o fator de maior prevalência, constituindo-se em 86,67\% dos casos. 0 paciente que utiliza dispositivo para fluidoterapia dispensa maior atenção ao dispositivo e isso pode fazer com que se distraia ao deambular, colocando-o em situação de risco para queda $^{(7)}$.

Outro fator que pode influenciar para um maior risco de quedas em pacientes em uso de terapia endovenosa é o fato de que a terapia endovenosa, muitas vezes, está associada à polifarmácia, podendo essa associação aumentar o risco para quedas, devido aos efeitos dos medicamentos, tais como ácido válproico, tramadol, furosemida, glifage, entre outros, que são comprovadamente relacionados a risco de quedas recorrentes, sendo denominados, potenciadores de quedas ${ }^{(19-22)}$. Ainda, os pacientes medicados com anti-hipertensivos apresentam maior risco de quedas, visto que, esses medicamentos podem provocar efeitos colaterais, tais como, hipotensão postural, tonturas e necessidade de urinar 
frequentemente, dentre outros efeitos, pode causar quedas e, consequentemente, fraturas ${ }^{(20)}$. Ainda, observa-se que as drogas associadas ao risco de quedas são amplamente utilizadas nos serviços de emergência.

Alteração do estado mental também foi apontada em outras pesquisas como fator de risco para quedas ${ }^{(16)}$. No presente estudo, cinco pacientes $(16,67 \%)$ esqueciam suas limitações ou superestimavam suas capacidades relacionadas à deambulação. Entende-se por superestima ou esquecimento de limitações, a situação em que o paciente é questionado quanto a sua necessidade de ajuda para ir ao banheiro sem auxílio e sua resposta é inconsistente ou não condiz com sua realidade ${ }^{(22)}$.

As alterações próprias do envelhecimento nos sistemas relacionados ao equilíbrio, a maior prevalência de doenças crônicas degenerativas e uso crônico e, por vezes, múltiplo de medicamentos, podem favorecer o aparecimento da tontura ou agravar o aparecimento desse sintoma, provocando maior limitação física, funcional e emocional, o que contribui para a ocorrência de quedas ${ }^{(22)}$.

Observa-se que os pacientes em observação, no serviço de emergência, estão sujeitos à alteração de comportamento, por suas condições clínicas agudizadas, alto fluxo de pessoal, além do ambiente desconhecido. Por essa razão, algumas vezes, os pacientes tentam sair do leito e sofrem quedas. Salienta-se que o risco de quedas se eleva, à medida que aumenta a coexistência desses fatores ${ }^{(12)}$.

A utilização de escala de avaliação para o risco de quedas padronizadas e adequadas ao perfil dos pacientes da instituição é uma exigência do Protocolo de Prevenção de Quedas. A utilização da MFS viabilizou a avaliação de pacientes internados no serviço de emergência, pois é de simples compreensão e de aplicação rápida, podendo ser usada na admissão do paciente e, diariamente, como é preconizado tanto pelo protocolo de prevenção de quedas do Ministério da Saúde quanto pela autora da escala $^{(1,22)}$.

A alta taxa de pacientes em observação no serviço de emergência com alto e médio risco de quedas, sugere a adoção de estratégias de prevenção como a utilização da avaliação do paciente para o risco de quedas e a prescrição de ações individuais ou coletivas para minimizar ou excluir os fatores de risco.

\section{CONCLUSÃO}

A aplicação da MFS possibilitou traçar um panorama dos fatores relacionados à queda nos pacientes que se encontravam em observação, no serviço de emergência da instituição, além de identificar um elevado risco para quedas.

Dentre os fatores de risco mais encontrados estão: a terapia endovenosa, o diagnóstico secundário e a marcha, o que representa um alerta para os enfermeiros, no que diz respeito ao diagnóstico precoce do risco de quedas, o planejamento e prescrição dos cuidados preventivos dispensados pela equipe de enfermagem, a esses pacientes, bem como o diálogo com a equipe de saúde como um todo.

A limitação deste estudo reside no fato da redução do número de leitos de internação disponíveis no período de coleta de dados, devido à reforma estrutural no referido serviço, ficando reduzido a três leitos de observação. $O$ estudo mostrou a necessidade de realização da avaliação do risco de quedas, diariamente, pelo enfermeiro, corroborando a necessidade de que essas anotações sejam evidenciadas no prontuário do paciente para que toda a equipe possa estar ciente do grau de risco do paciente.

A existência de poucos estudos publicados sobre quedas em serviços de emergência dificultou a comparação com outros casos e evidenciou a importância de pesquisas sobre o tema. Comprova-se a necessidade de implantação de protocolos de prevenção de quedas no serviço de emergência e a realização da avaliação do risco de quedas, diariamente, pelo enfermeiro como uma ferramenta para garantir um cuidado seguro.

\section{REFERÊNCIAS}

1- Brasil. Protocolo prevenção de quedas. Brasília: Ministério da Saúde; 2013 [citado em 14 out 2018]. Disponível em: https://www20.anvisa.gov.br/segurancadopacien te/index.php/publicacoes/item/prevencao-dequedas

2- World Health Organization (WHO). World alliance for patient safety: Forward programme 2008-2009]. Geneva: Who; 2008.

3- Duarte SCM, Stipp MAC, Silva MM, Oliveira FT. Eventos adversos e segurança na assistência de enfermagem. Rev Bras Enferm. 2015;68(1):14454. DOI: $10.1590 / 0034-7167.2015680120 p$

4- Morsch P, Myskiw M, Myskiw JC. A problematização da queda e a identificação dos 
fatores de risco na narrativa de idosos. Ciênc Saúde Coletiva 2016;21(11):3565-74. DOI: 10.1590/1413-812320152111.06782016

5- Vaccari E, Lenardt $\mathrm{MH}$, Willig $\mathrm{MH}$, Betiolli SE, Andrade LAS. Segurança do paciente idoso e o evento de queda no ambiente hospitalar. Cogitare Enferm. 2016;21(nesp):1-9. DOI: 10.5380/ce.v21i5.45562

6- Pasa TS, Magnago TSBS, Urbanetto JS, Baratto MAM, Moraes BX, Carollo JB. Risk assessment and incidence of falls in adult hospitalized patients. Rev Latino-Am Enfermagem 2017;25:1-8. DOI: 10.1590/15188345.1551.2862

7- Urbanetto JS, Creutzberg M, Franz F, Ojeda BS, Gustavo AS, Bittencourt HR, et al. Morse fall scale: Translation and transcultural adaptation for the portuguese language [internet] Rev Esc Enferm USP 2013;47(3):569-75. DOI: 10.1590/S0080-623420130000300007

8- Polit DF, Beck CT, Hungler BP. Fundamentos de pesquisa em enfermagem: Métodos, avaliação e utilização. 5a ed. Porto Alegre: Artes Médicas; 2004.

9- Lacerda MR, Costenaro RGS. Metodologias da pesquisa para enfermagem e saúde: Da teoria a prática. Porto Alegre: Moriá; 2016.

10- Brasil. Ministério da Saúde. Envelhecimento e saúde da pessoa idosa. Brasília: Ministério da Saúde; 2006.

11- Brasil. Conselho Nacional de Saúde. Resolução no 466, de 12 de dezembro de 2012. Dispõe sobre as diretrizes e as normas regulamentadoras de pesquisa envolvendo seres humanos. Diário Oficial da União 2012.

12- Ashley S, Iseler JI, Havey R, Aebersold C, Oak $R$, Lansing E. Catching quality before it falls: Preventing falls and injuries in the adult emergency department. J Emerg Nurs. 2019;45(3):257-64.

DOI:

10.1016/j.jen.2018.08.001

13- Sarges NA, Santos MPAO, Chaves EC. Avaliação da segurança do idoso hospitalizado quanto ao risco de quedas. Rev Bras Enferm. 2017;70(4):896-903. DOI: 10.1590/0034-71672017-0098

14- Custódio EB, Malaquias Júnior J, Callil Voos M. Relação entre cognição (função executiva e percepção espacial) e equilíbrio de idosos de baixa escolaridade. Fisioter Pesqui. 2010;17(1):46-51. DOI: 10.1590/S1809$\underline{29502010000100009}$

15- Nascimento ERP, Silva SG, Souza BC, Souza DD, Germer Netto A. Ambiência da emergência ao cuidado do idoso. Esc Anna Nery 2015;19(2): 338-42. DOI: 10.5935/1414-8145.20150046 16- Silva AT, Alves MG, Sanches RS, Terra FS, Resck ZMR. Assistência de enfermagem e o enfoque da segurança do paciente no cenário brasileiro [internet] Saúde Debate 2016;40(111): 292-301. DOI: 10.1590/0103-1104201611123

17- Morse JM. Preventing patient falls. 2th ed. New York: Springer; 2009.

18- Schwendimann R, Geest S, Milisen K. Evaluation of the Morse Fall Scale in hospitalised patients. Age Ageing 2006;35(3):311-3. DOI: 10.1093/ageing/afj066

19- Reis KMC, Jesus CAC. Relação da polifarmácia e polipatologia com a queda de idosos institucionalizados. Texto Contexto Enferm. 2017;6(2):1-9. DOI: 10.1590/010407072017003040015

20- Alves RLT, Silva CFM, Pimentel LM, Costa IA, Souza ACS, Coelho LAF. Avaliação dos fatores de risco que contribuem para quedas em idosos. Rev Bras Geriatr Gerontol. 2017;20(1):59-69. DOI: 10.1590/1981-22562017020.160022

21- Luzia MF, Victor MAG, Lucena AF. Diagnóstico de enfermagem Risco de quedas: Prevalência e perfil clínico de pacientes hospitalizados. Rev Latino-Am Enfermagem 2014;22(2):262-8. DOI: $10.1590 / 0104-$ 1169.3250 .2411

22- Rodrigues IG, Fraga GP, Barros MBA. Quedas em idosos: Fatores associados em estudo de base populacional. Rev Bras Epidemiol. 2014;17(3):705-18. DOI: $\quad$ 10.1590/18094503201400030011

Nota: Não houve suporte financeiro de agência de fomento. Este artigo é resultado do trabalho final de residência em enfermagem.

Recebido em: 04/01/2019

Aprovado em: 24/06/2019

Endereço de correspondência:

Paloma Horbach da Rosa

Travessa Cassel, 290. Nossa Senhora de Lourdes.

CEP: 97050-110 - Santa Maria/RS - Brasil

E- mail: palomahorbach93@hotmail.com 